\title{
ESTUDOS SOBRE O ENSINO E APRENDIZAGEM DE CONCEITOS EM ELETROQUÍMICA: UMA REVISÃO
}

\author{
STUDIES ON TEACHING AND LEARNING OF CONCEPTS IN ELECTROCHEMISTRY: \\ A REVIEW
}

Danielle de Oliveira Vieira ${ }^{1}$, Marcel Bruno Pereira Braga ${ }^{2}$, Raimundo Ribeiro Passos ${ }^{3}$, Sidilene de Aquino Farias ${ }^{4}$

Recebido: abril/2020 Aprovado: março/2021

\begin{abstract}
Resumo: O presente estudo objetivou mapear e classificar, nas pesquisas que abordam o ensino e aprendizagem de conceitos eletroquímicos, aspectos metodológicos, base teórica e principais contribuições para a área de Ensino de Química no Brasil. Para isso, utilizou-se os seguintes procedimentos de coleta de dados: (1) seleção dos artigos; (2) triagem a partir da leitura integral dos artigos; (3) análise dos artigos baseada nas seguintes categorias de análise: Aspectos Metodológicos; Base Teórica e Resultados das Pesquisas. Foram selecionados 19 artigos entre quatro periódicos selecionados, cujos resultados obtidos revelam que a maioria das pesquisas é empírica, com abordagem qualitativa e prioriza o Ensino Médio e Ensino Superior. Em relação às Bases Teóricas, as pesquisas pautam-se em teorias Cognitivistas - Teoria da Aprendizagem Significativa e Teoria Sociocultural, fundamentam-se também no campo da Didática das Ciências, sendo predominante a experimentação (demonstrativa, investigativa e/ou problematizadora). Por fim, os Resultados das Pesquisas apontam as dificuldades de aprendizagem e estratégias remediativas das mesmas, destacando-se que a utilização de estratégias remediativas perpassa pela superação de visões simplistas das concepções de ciências, da natureza da ciência e de concepções de ensino e aprendizagem, tanto na formação inicial e continuada de professores de Ciências.
\end{abstract}

Palavras-chave: conceitos eletroquímicos, dificuldades de aprendizagem, ensino-aprendizagem.

Abstract: This study aimed at mapping and classifying research about the teaching and learning of electrochemical concepts, methodological aspects, theoretical basis in addition to the main contributions to teaching and learning of Chemistry in Brazil. To this end, the study used the following procedures to generate the data: (1) selection of articles; (2) screening relevant articles based on the reading of full articles; (3) analysis of articles based on two categories: Methodological Aspects and Theoretical Base and Results of Research. Nineteen articles from four journals were selected which results reveal that most research is empirical, carried out according to qualitative approaches and emphasize High School and Higher Education. Concerning the Theoretical Bases, the research analyzed were supported in Cognitivist theories - Meaningful Learning Theory and Sociocultural Theory; the research is also supported by Science Didactics in which experimentation (demonstrative, investigative and/or problem-solving activities) prevail. This study demonstrates the result of the analyzed research point out the learning difficulties and remedial strategies to overcome their own limitations, highlighting that the use of remedial strategies involves overcoming simplistic visions of concepts of sciences, of the nature of science

1 iD https://orcid.org/0000-0001-8309-7917- Mestre em Engenharia (UFAM). Doutoranda (UFAM), Manaus, Amazonas, Brasil. Rua Atagamita, 190B, Aleixo, 69060050, Manaus, AM, Brasil).E-mail: danivieiralq@gmail.com

2 iD https://orcid.org/0000-0003-2226-1538 - Doutorado em Doutoramento em Ensino das Ciências (UC). Professor (UFAM), Manaus, Amazonas, Brasil. Av. Gal. Rodrigo Otávio Jordão Ramos, 6200 - Bloco 10 - Setor Norte, Coroado. E-mail: marcelbruno79@hotmail.com

3 iD https://orcid.org/0000-0002-5478-9604 - Doutorado em Fisico-Química (USP). Professor (UFAM), Manaus, Amazonas, Brasil. Av. Gal. Rodrigo Otávio Jordão Ramos, 6200 - Bloco 10 - Setor Norte, Coroado. E-mail: rrpassos@ufam.edu.br

4 (D) https://orcid.org/0000-0003-3866-207X - Doutorado em Química (UFSCAR). Professora (UFAM), Manaus, Amazonas, Brasil. Av. Gal. Rodrigo Otávio Jordão Ramos, 6200 - Bloco 10 - Setor Norte, Coroado. E-mail: sfarias@ufam.edu.br 
and the conceptions of teaching and learning, both in initial and continuing education of Science teachers.

Keywords: electrochemical concepts, learning disabilities, teaching-learning.

\section{Introdução}

A aprendizagem está relacionada à necessidade que o ser humano tem de compreender o mundo no qual está inserido e de melhorar a forma de se relacionar com ele. Assim, é importante compreender e identificar evidências e características de uma boa aprendizagem, para que se possam propor meios para o seu desenvolvimento e aquisição de conhecimentos.

Dentre as várias definições de aprendizagem nos mais diversos domínios, existem aspectos comuns que podem favorecer uma boa aprendizagem, como: (1) a produção de uma mudança duradoura; (2) transferência para novas situações; (3) consequência direta na prática realizada (POZO, 2002). Contudo, pesquisas têm apontado que o desenvolvimento da aprendizagem nem sempre ocorre de forma satisfatória. Lima e Leite (2012) investigaram o baixo nível de aprendizagem e as causas do desinteresse de estudantes e, verificaram que entre as principais causas encontram-se a falta de contextualização do conteúdo ensinado e a falta de preparo dos professores. FRISON e DEL PINO (2015) ao questionarem os estudantes sobre quando se tornava fácil aprenderem ciência verificaram que a maioria deles respondeu se relacionando ao conteúdo e a metodologia utilizada pelo professor nas aulas. Uehara (2005) em estudo sobre erros conceituais cometidos por estudante durante a abordagem do tema Equilíbrio Químico considera como causas dessas dificuldades o tratamento superficial do conteúdo tanto pelo professor como pelo livro didático, o próprio material didático utilizado, aulas extremamente teóricas e as ideias prévias dos estudantes. Acrescente-se ainda que, o aprendizado de conceitos com os quais os estudantes não estão familiarizados, o nível de abstração e a linguagem empregada tornam difícil a assimilação dos conteúdos ministrados e, além disso, a simplificação dos conteúdos com o intuito de facilitar o aprendizado é prejudicial (BERLOTTI, 2011).

Outro elemento que interfere na aprendizagem de conceitos científicos, diz respeito às concepções que os estudantes já possuem sobre alguns fenômenos (ROSA; SCHNETZLER, 1998). Tais concepções, sendo distantes da visão cientificamente aceita tornam-se acentuadas devido: (1) à ausência de discussões nas aulas de Ciências; (2) à ênfase na visão empiricista do conhecimento; e, (3) à falta de relacionamento explícito entre os níveis micro e macroscópico do conhecimento químico no Ensino Tradicional. Também pode-se mencionar, a falta de valorização das relações existentes entre teorias e modelos científicos, que tentam explicar a natureza da matéria e os fenômenos observáveis. Isto porque os estudantes têm que articular dois mundos muito diferentes: o macroscópico e o microscópico por meio de uma linguagem simbólica.

No universo da disciplina Química, o conteúdo Eletroquímica está entre os mais difíceis de ensinar e de aprender (NOGUEIRA; GOES; FERNANDEZ, 2017). Os conceitos abordados em Eletroquímica são considerados abstratos e assim de difícil compreensão pelos alunos, como por exemplo, na determinação dos polos de uma pilha (MATSUBARA; NERI; ROSOLEN, 2007), ou a compreensão de reações químicas e reações redox (MORTIMER; MIRANDA, 1995; JUSTI, 
1998), além da abordagem inadequada destes conceitos nos Livros didáticos (MENDONÇA; CAMPOS; JÓFILI, 2004; PITANGA et al., 2014).

$E$, na tentativa de superar estas dificuldades de aprendizagem dos conceitos científicos, encontram-se várias pesquisas propondo estratégias didáticas para o ensino-aprendizagem de eletroquímica tais como: o uso de experimentos investigativos envolvendo a observação de fenômenos cotidianos (MERÇON; GUIMARÃES; MAINIER, 2004, 2011; WARTHA et al., 2007; SARTORI; BATISTA; FATIBELLO-FILHO; 2008; SARTORI et al., 2013; RODRÍGUEZ; PÉREZ, 2016; WARTHA; LEMOS, 2016; MATHIAS; SILVA P.; SILVA O., 2017); o uso de experimentos demonstrativos envolvendo óxido-redução (FRANCISCO JUNIOR; DOCHI, 2006), utilização de modelos físicos como auxílio na formação de imagens mentais (FRANCISCO JUNIOR; FERREIRA; HARTWIG, 2009); a importância da contextualização na abordagem de conceitos eletroquímicos (LIMA; MERÇON, 2011; KLEIN; BRAIBANTE, 2017) e a valorização do contexto histórico na aquisição de conceitos (GERMANO; LIMA; SILVA , 2012).

Diante disso, para este texto foram selecionadas cinco revistas conforme a classificação do Qualis A1 a B5, na avaliação 2013 a 2016 de acordo com a Coordenação de Aperfeiçoamento de Pessoal de Nível Superior (Capes). Assim, apresentamos uma análise da produção científica presente em revistas nacionais e estrangeiras, sendo: Enseñanza de las Ciencias: Investigaciones Didácticas, Química Nova na Escola, Química Nova, Revista Electrónica de Enseñanza de las Ciências e Caderno Brasileiro de Ensino de Física, a respeito da aprendizagem de conceitos em Eletroquímica, desde a primeira edição até a edição de 2018. A partir desta análise, pretendese responder aos seguintes questionamentos:

a) Qual o perfil metodológico das pesquisas selecionadas neste estudo?

b) Quais os pressupostos teóricos assumidos pelos autores das referidas pesquisas e sua pertinência para a própria pesquisa?

c) Quais as principais contribuições das pesquisas para a compreensão da aprendizagem de conceitos eletroquímicos?

d) O que os estudos apresentam sobre aprendizagem de conceitos eletroquímicos?

e) Quais as dificuldades encontradas na aprendizagem de conceitos eletroquímicos?

f) Quais as estratégias utilizadas para facilitar a aprendizagem de conceitos eletroquímicos?

A partir desses questionamentos, propõe-se o seguinte objetivo: mapear e classificar os aspectos metodológicos, a base teórica e as principais contribuições destes estudos para a área de Ensino de Química no Brasil.

\section{Caminho Metodológico}

Neste trabalho utiliza-se um desenho metodológico pautado no enfoque misto de pesquisa científica com preponderância do enfoque Qualitativo (QUAL-quan) (Figura 1). Essa combinação de abordagens quantitativas e qualitativas de pesquisa visa a complementariedade entre métodos como forma de ter uma visão mais ampla e profunda do objeto de estudo. De 
modo geral, a abordagem quantitativa de pesquisa pauta-se em medidas e observações de um conjunto de variáveis controladas por meio de projeto e análise estatística. Enquanto na abordagem qualitativa, o pesquisador realiza a coleta de dados emergentes abertos no intuito de produzir determinados temas (CRESWELL, 2007).

Iniciando a Etapa com ênfase na recolha dos dados (Figura 1), foram estabelecidos os seguintes critérios de inclusão/exclusão dos periódicos: (1) periódicos relacionados à Área Química que tenham Qualis A1 a B5 na avaliação 2013 a 2016, de acordo com a Coordenação de Aperfeiçoamento de Pessoal de Nível Superior (Capes); (2) periódicos da área de Ensino que tenham Qualis A1 e A2 na avaliação 2013 a 2016; (3) periódicos que o escopo permite a abordagem de conceitos químicos, na perspectiva do processo de ensino e aprendizagem. Cumpre esclarecer que, tais critérios foram determinados visto que este artigo é um recorte de uma pesquisa de doutorado, em desenvolvimento, que estuda a aprendizagem de conceitos em Eletroquímica de estudantes do ensino médio.

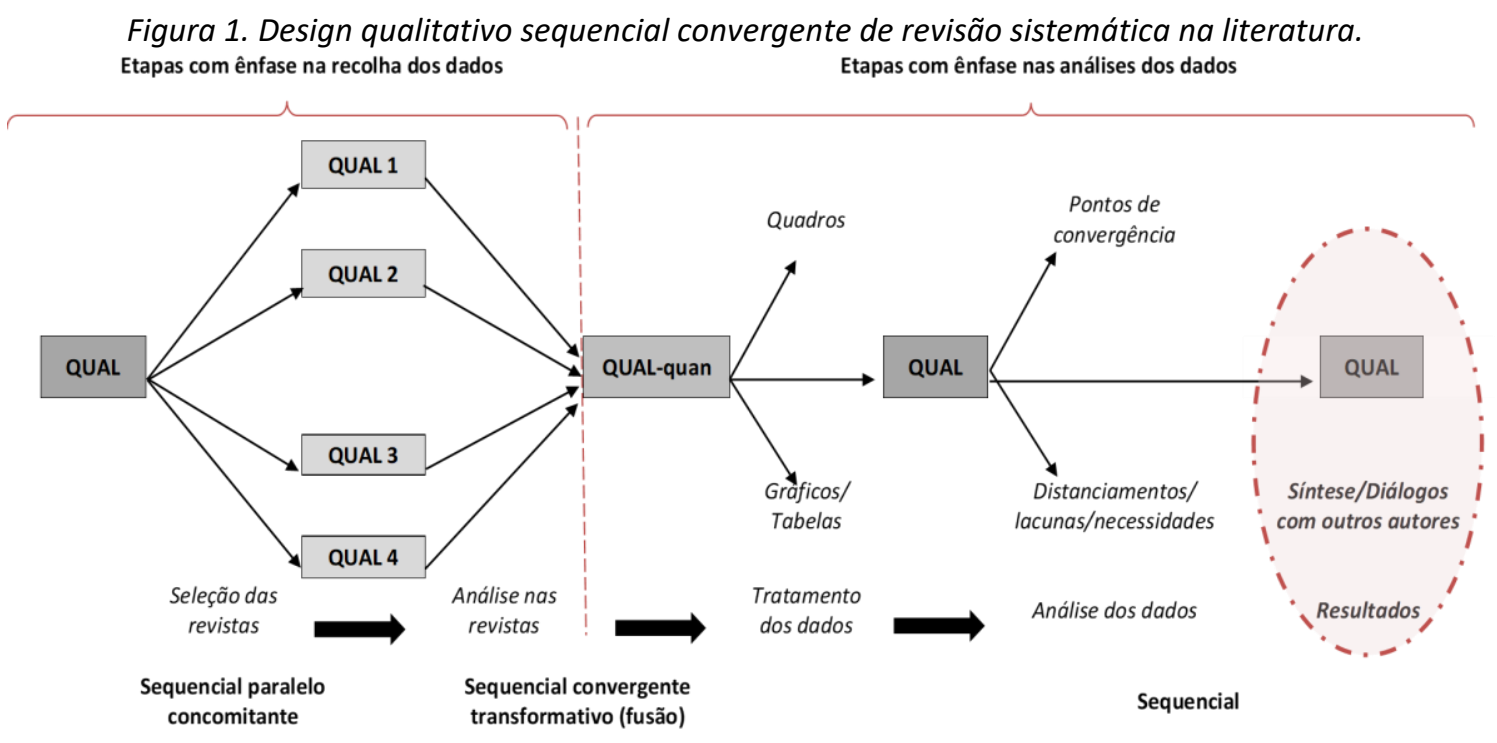

Legenda: QUAL 1 - Enseñanza de las Ciencias: Investigaciones Didácticas; QUAL 2 - Química Nova na Escola; QUAL 3 - Revista Electrónica de Enseñanza de las Ciencias e QUAL 4 - Caderno Brasileiro de Ensino de Física.

Fonte: elaborada pelos autores.

Dessa maneira, realizou-se o levantamento em cinco periódicos: revista Enseñanza de las Ciencias: Investigaciones Didácticas, destinada a professores e pesquisadores no campo do Ensino de Ciências e Matemática; revista Química Nova, que publica artigos com resultados originais de pesquisa, trabalhos de revisão, divulgação de novos métodos e técnicas, educação e assuntos gerais na área de química; revista Química Nova na Escola, destinada à comunidade de Educação Química; Revista Electrónica de Enseñanza de las Ciencias, dedicada à inovação e investigação sobre o ensino e a aprendizagem das ciências experimentais nos níveis infantil, primário, secundário e universitário; Revista Caderno Brasileiro de Ensino de Física, voltada para os cursos de formação de professores de Física (Quadro 1).

Nessa etapa foram utilizadas ferramentas de busca, utilizando termos como: aprendizagem, concepções alternativas, eletroquímica, ânodo, cátodo, pilhas, eletrólise, reações químicas, redox, oxirredução, ponte salina, Nox (número de oxidação), íon, cátion, 
ânion, voltagem, ddp (diferença de potencial), corrosão, eletrodo, redução, oxidação, oxidante, redutor, solução eletrolítica e condutividade elétrica. Então, foi realizada a leitura dos títulos, resumos e palavras-chave, nos artigos selecionados, iniciando-se a Etapa com ênfase na análise dos dados.

Foram encontrados um total de 156 artigos, sendo 31 artigos na QUAL 1, 48 artigos na QUAL 2; 54 artigos na QUAL 3, 14 artigos na QUAL 4 (Figura 1). Também foram encontrados 9 artigos na revista Química Nova, sendo descartados artigos cujo desenvolvimento limitava-se ao ensino de conceitos químicos em geral (Ex. reações químicas, transformações) e que não apresentavam dados sobre aprendizagem de conceitos eletroquímicos. Outro critério de exclusão foi relacionado a propostas de ensino que não versavam sobre aprendizagem em Eletroquímica. Diante dessa triagem, não foram selecionados artigos da revista Química Nova para o tratamento dos dados.

Quadro 1. Revistas nas quais foi realizada a Revisão de Literatura sobre aprendizagem de conceitos eletroquímicos.

\begin{tabular}{cccc}
\hline \hline CÓDIGO & REVISTA & INÍCIO & $\begin{array}{c}\text { PERIODICIDADE } \\
\text { ATUAL }\end{array}$ \\
\hline \hline QUAL 1 & Enseñanza de las Ciencias: Investigaciones Didácticas & 1983 & Trimestral \\
\hline QUAL 2 & Química Nova na Escola & 1995 & Quadrimestral \\
\hline QUAL 3 & Revista Electrónica de Enseñanza de las Ciencias & 2002 & Trimestral \\
\hline QUAL 4 & Caderno Brasileiro de Física & 1984 & Quadrimestral \\
\hline & Fonte: Elaborada pelos autores. &
\end{tabular}

Após a análise nas revistas, selecionado os artigos, foi realizada a leitura integral e minuciosa de cada artigo, com a finalidade de identificar, quantificar, analisar e descrever padrões e tendências (QUAL-quan) sobre a produção do conhecimento científico em relação ao Ensino e Aprendizagem de Conceitos Eletroquímicos. Essa leitura foi realizada sobre o resumo, a fundamentação teórica, a metodologia e da discussão dos resultados. A partir disso, selecionou-se aqueles artigos que versavam sobre a aprendizagem, a aquisição de conceitos e/ou apresentavam contribuições para a aprendizagem de conceitos eletroquímicos na sala de aula. Dessa forma, após esta triagem foram selecionadas 19 publicações que versavam sobre a temática em estudo (Quadro 2). Os dados obtidos foram organizados em tabelas, gráficos e quadros.

A Análise dos dados por meio da técnica de Análise Textual Discursiva, segundo Moraes e Galiazzi (2007), foi o momento em que foram elaboradas e refinadas categorias de análise, a partir da intepretação das regularidades, padrões e tópicos presentes nos dados recolhidos, buscando-se pontos de convergências, distanciamentos, lacunas e necessidades. Assim, a análise envolveu organização dos dados, a divisão em unidades manipuláveis, síntese e procura de padrões. Os artigos foram lidos e examinados individualmente considerando-se os seguintes temas: Aspectos metodológicos, Base teórica e Resultados das Pesquisas.

Nos Aspectos Metodológicos, descreve-se o perfil metodológico dos estudos levantados, foram analisados por meio de categorias já descritas na literatura (COSTA; MOREIRA, 1996; HOPPEN; MEIRELES, 2005), tais como: (i) Natureza da pesquisa; (ii) Abordagem da pesquisa; (iii) Objetivo da pesquisa (iv) Nível de ensino. Na categoria Natureza da Pesquisa, classificaram-se 
os estudos em teórico ou empírico. As pesquisas teóricas consistem na re-construção de teorias, conceitos e no aprimoramento dos fundamentos teóricos (DEMO, 2000; OLIVEIRA, 2007). Quanto a isso, vale dizer que nas pesquisas nem sempre se encontram reconstruções teóricas, conhecimentos novos, sendo mais comum as reproduções teóricas, ou seja, pesquisas que se utilizam dos embasamentos teóricos, contribuindo na re-construção de condições explicativas de dada realidade e de discussões pertinentes, exemplo, pesquisa bibliográfica e pesquisa documental. Enquanto, as pesquisas empíricas referem-se ao tratamento experimental da realidade, produzindo e analisando dados que conferem maior concretude às argumentações, devido sua aproximação à prática (CHIZZOTTI, 2018).

A categoria Abordagem da Pesquisa consiste nos métodos empregados nos artigos para coleta e análise dos dados. Dessa forma, as publicações foram classificadas em: pesquisa qualitativa, quando envolvem análises descritivas que enfatizam a subjetividade do sujeito e tendo o ambiente natural como sua fonte de dados; pesquisa quantitativa, a qual mensura dados, valendo-se de métodos estatísticos e buscando generalização dos resultados a uma certa população de estudo e pesquisa mista, a que envolve tanto os aspectos qualitativos e quanto os quantitativos (FONSECA, 2002; CRESWELL, 2007; FLICK, 2009).

Segundo Gil (2008), um estudo pode ser classificado quanto ao Objetivo da Pesquisa em três grandes grupos, sendo eles: pesquisa exploratória, pesquisa descritiva e pesquisa explicativa. A pesquisa exploratória objetiva a maior familiaridade com o problema de pesquisa, visando torná-lo mais explícito ou mesmo possibilitar a construção de hipóteses. Em sua maioria são levantamentos bibliográficos; entrevistas com pessoas que tiveram experiências práticas com o problema e a análise de exemplos que estimulam a compreensão. Por isso, elas podem ser classificadas em pesquisa bibliográfica e estudo de caso (GIL, 2008). As pesquisas descritivas tal como as exploratórias, são as mais utilizadas pelos pesquisadores sociais atentos à atuação prática. Elas "têm como objetivo primordial a descrição das características de determinada população ou fenômeno ou, então, o estabelecimento de relações entre variáveis" (GIL, 2002, p.42).

São inumeráveis as pesquisas assim classificadas e as características mais significativas estão na utilização de técnicas padronizadas de coleta de dados, como questionário e observação sistemática (GIL, 2002; 2008). São exemplos de pesquisa descritiva: estudos de caso, análise documental e pesquisa ex-post-facto (GERHARDT; SILVEIRA, 2009). Enquanto pesquisa explicativa ocupa-se na identificação dos fatores que determinam ou que contribuem para a ocorrência dos fenômenos. Ou seja, atem-se na explicação do porquê das coisas por meio dos resultados oferecidos. A pesquisa explicativa pode ser a continuação da pesquisa descritiva, visto que a identificação de fatores que determinam um fenômeno requer que ele seja detalhadamente descrito. Pesquisas desse tipo podem ser classificadas como experimentais e ex-post facto (GIL, 2008).

Na categoria Nível de Ensino, organizou-se as pesquisas conforme as etapas da Educação Brasileira, sendo as mesmas: Ensino Fundamental, Ensino Médio e Educação Superior.

No tema Base Teórica foi identificada a fundamentação dos artigos analisados. Tal análise torna-se importante por permitir a avaliação dos pressupostos teóricos assumidos pelos autores 
das referidas pesquisas, bem como sua pertinência para a pesquisa desenvolvida (ALVESMAZZOTTI; GEWANDSZNAJDER, 1999). Nesse critério as categorias emergiram a posteriori. Em relação ao tema Resultados das Pesquisas, analisam-se as contribuições dos artigos avaliados para a Aprendizagem de Conceitos Eletroquímicos. As categorias desse tema são emergentes ( $a$ posteriori).

Quadro 2. Artigos publicados que abordam ensino e aprendizagem de conceitos eletroquímicos.

\begin{tabular}{|c|c|c|c|}
\hline REVISTA & ANO & AUTORES & TÍTULO \\
\hline QUAL 1 & 2008 & $\begin{array}{l}\text { GUISASOLA, J.; ZUBIMENDI, J. L.; } \\
\text { ALMUDÍ, J. M.; CEBERIO, M. }\end{array}$ & $\begin{array}{l}\text { Dificultades persistentes en el aprendizaje } \\
\text { de la electricidad: estrategias de } \\
\text { razonamiento de los estudiantes al explicar } \\
\text { fenómenos de carga eléctrica. }\end{array}$ \\
\hline \multirow{7}{*}{ QUAL 2} & 1996 & BOFF, E. T. de O.; FRISON, M. D. & $\begin{array}{l}\text { Explorando a existência de cargas elétricas } \\
\text { na matéria }\end{array}$ \\
\hline & 1996 & LOPES, A. R. C. & $\begin{array}{llll}\text { Potencial de } & \text { Redução } & \text { e } \\
\text { eletronegatividade, obstáculo verbal. } & \end{array}$ \\
\hline & 2009 & $\begin{array}{l}\text { SANJUAN, M. E. C.; SANTOS, C. V. dos; } \\
\text { MAIA, J. de O.; SILVA, A. F. A. da; } \\
\text { WARTHA, E. J. }\end{array}$ & $\begin{array}{l}\text { Maresia: Uma Proposta para o Ensino de } \\
\text { Eletroquímica }\end{array}$ \\
\hline & 2011 & $\begin{array}{l}\text { FRAGAL, V. H.; MAEDA, S. M.; PALMA, } \\
\begin{array}{ll}\text { E. P. da; BUZATTO, M. B. P.; } \\
\text { RODRIGUES, R. A.; SILVA, E. L. }\end{array}\end{array}$ & $\begin{array}{l}\text { Uma proposta alternativa para o ensino de } \\
\text { eletroquímica sobre a reatividade de } \\
\text { metais }\end{array}$ \\
\hline & 2016 & $\begin{array}{l}\text { SILVA, R. M. da; SILVA, R. C. da; } \\
\text { ALMEIDA, M. G. O. de; AQUINO, K. A. } \\
\text { da S. }\end{array}$ & $\begin{array}{l}\text { Conexões entre } \\
\text { Eletrótica Química e } \\
\text { Elequica: A Experimentação na } \\
\text { Perspectiva de Uma Aprendizagem } \\
\text { Significativa }\end{array}$ \\
\hline & 2017 & $\begin{array}{l}\text { BARRETO, B. S. J.; BATISTA, C. H.; } \\
\text { CRUZ, M. C. P. }\end{array}$ & $\begin{array}{l}\text { Células Eletroquímicas, Cotidiano e } \\
\text { Concepções dos Educandos }\end{array}$ \\
\hline & 2018 & $\begin{array}{l}\text { SANTOS, T. N. P.; BATISTA, C. H.; } \\
\text { OLIVEIRA, A. P. C.; CRUZ, M. C. P. }\end{array}$ & $\begin{array}{l}\text { Aprendizagem Ativo-Colaborativo- } \\
\text { Interativa: Inter-Relações } \\
\text { Experimentação Investigativa no Ensino de } \\
\text { Eletroquímica }\end{array}$ \\
\hline \multirow{5}{*}{ QUAL 3} & 2008 & $\begin{array}{l}\text { COSTA, A. R. da; OLIVEIRA, J. P.; } \\
\text { ALVES, J. M. }\end{array}$ & $\begin{array}{l}\text { Analisando a construção de explicações } \\
\text { individuais e coletivas em aulas sobre } \\
\text { ligações iônicas, na 8a série. }\end{array}$ \\
\hline & 2012 & $\begin{array}{l}\text { CÁSSIO, F. L.; CORDEIRO, D. de S.; } \\
\text { CORIO, P.; FERNANDEZ, C. }\end{array}$ & $\begin{array}{l}\text { O protagonismo subestimado dos íons nas } \\
\text { transformações químicas em solução por } \\
\text { livros didáticos e estudantes de química }\end{array}$ \\
\hline & 2013 & FRANCISCO JUNIOR, W. & $\begin{array}{l}\text { El uso de un caso de investigación para el } \\
\text { estudio de los métodos electrolíticos: Una } \\
\text { experiencia en la educación superior }\end{array}$ \\
\hline & 2014 & COELHO, G. R.; AMANTES, A. & $\begin{array}{l}\text { A influência do engajamento sobre a } \\
\text { evolução do entendimento dos estudantes } \\
\text { em eletricidade }\end{array}$ \\
\hline & 2016 & $\begin{array}{l}\text { SANTOS JUNIOR, J. B.; BENEDETTI } \\
\text { FILHO, E.; CAVAGIS, A. D. M.; } \\
\text { ANUNCIAÇÃO, E. A. }\end{array}$ & 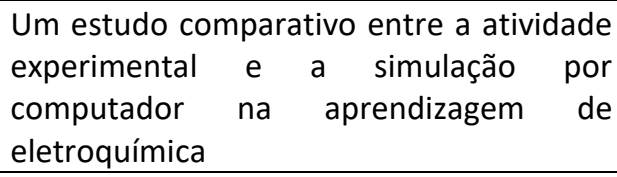 \\
\hline \multirow[t]{2}{*}{ QUAL 4} & 1986 & $\begin{array}{l}\text { VIEIRA, J. S.; QUILLFELDT, J. A.; } \\
\text { SELISTRE, L. F.; RIOS, L. H. R.; } \\
\text { SCHMITZ, S. R.; STEFFANI, M. H. }\end{array}$ & $\begin{array}{l}\text { Conservação de corrente elétrica num } \\
\text { circuito elementar: o que os alunos } \\
\text { pensam a respeito? }\end{array}$ \\
\hline & 1994 & AXT, R.; ALVES, V. M. & $\begin{array}{l}\text { O papel do voltímetro na aquisição do } \\
\text { conceito de diferença de potencial }\end{array}$ \\
\hline
\end{tabular}




\begin{tabular}{lll}
\hline 2003 & $\begin{array}{l}\text { PACCA, J. L. A.; FUKUI, A.; BUENO, M. } \\
\text { C. F.; COSTA, R. H. P.; VALÉRIO, R. M.; } \\
\text { MANCINI, S. }\end{array}$ & $\begin{array}{l}\text { Corrente elétrica e circuito elétrico: } \\
\text { algumas concepções do senso comum }\end{array}$ \\
\hline 2009 & $\begin{array}{l}\text { LABURÚ, C. E.; GOUVEIA, A. A.; } \\
\text { BARROS, M. A. }\end{array}$ & $\begin{array}{l}\text { Estudo de circuitos elétricos por meio de } \\
\text { desenhos dos alunos: uma estratégia } \\
\text { pedagógica para explicitar as dificuldades } \\
\text { conceituais }\end{array}$ \\
\hline & CARAMEL, N. J. C.; PACCA, J. L. A. & $\begin{array}{l}\text { Concepções alternativas em eletroquímica } \\
\text { e circulação da corrente elétrica. }\end{array}$ \\
\hline & $\begin{array}{l}\text { Las actividades de laboratorio en la } \\
\text { VILLAFUERTE, M. }\end{array}$ & $\begin{array}{l}\text { formación de ingenieros: propuesta para } \\
\text { aprendizaje de los fenómenos de } \\
\text { conducción eléctrica. }\end{array}$ \\
\hline
\end{tabular}

Fonte: Elaborada pelos autores.

\section{Resultados e Discussão}

Conforme mencionado anteriormente, foram encontrados 19 artigos que abordam aspectos relacionados ao ensino e aprendizagem de conceitos eletroquímicos. O levantamento dos artigos foi realizado para cada revista, desde seu primeiro número disponível online até o ano de 2018. A análise dos dados a partir dos critérios utilizados permitiu a discussão quanto à caracterização de informações básicas, aos aspectos metodológicos, à base teórica e aos resultados presentes nas pesquisas analisadas.

\subsection{Caracterização de Informações Básicas}

Buscou-se fazer uma caracterização de informações básicas para o conjunto de artigos que foram encontrados sobre o objeto de investigação deste trabalho, aprendizagem de conceitos eletroquímicos. Observou-se que as quantidades de artigos por revista foi a seguinte: QUAL 1 - um artigo; QUAL 2 - sete artigos; QUAL 3 - cinco artigos; QUAL 4 - seis artigos. No que tange à distribuição dessas pesquisas por período, foi possível verificar que nas décadas de $80 \mathrm{e}$ 90 poucos trabalhos foram realizados sobre o ensino de conceitos em eletroquímica (Figura. 2), sendo uma maior concentração de trabalhos nos períodos de 2001 a 2018. Também foi observado que dois pesquisadores estão presentes em mais de um trabalho, caracterizando pesquisas desenvolvidas por um mesmo grupo de pesquisa.

Figura 2. Distribuição por período do conjunto de artigos encontrados sobre ensino e aprendizagem de conceitos em eletroquímica.

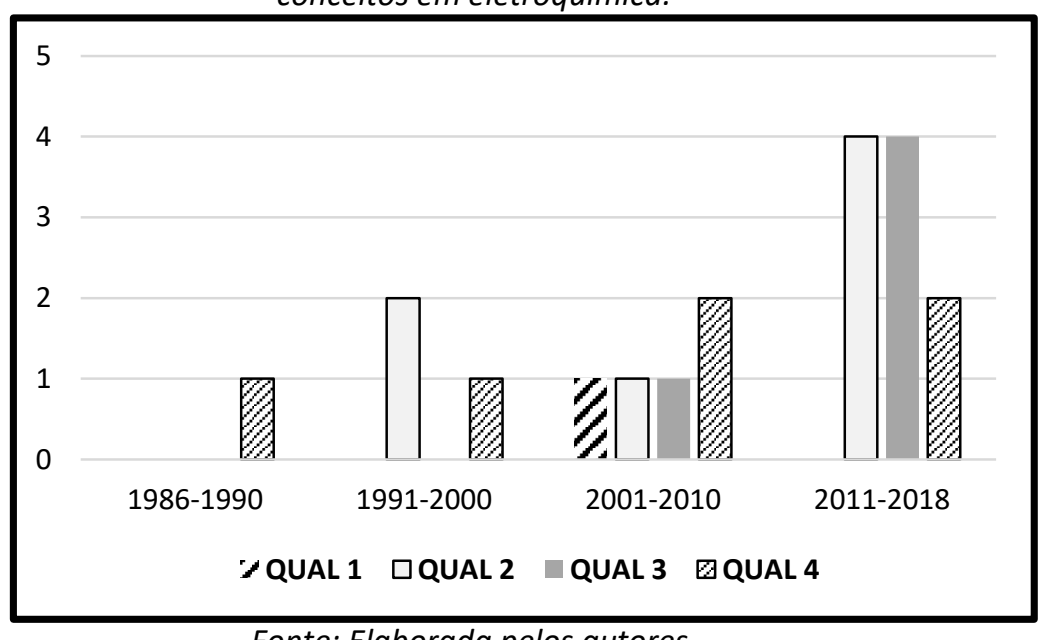

Fonte: Elaborada pelos autores. 
Observa-se assim que surgiram ao longo deste tempo maior número de estudos sobre aprendizagem de conceitos em eletroquímica. Sendo o número mais expressivo na QUAL 2 e na QUAL 3. Destacamos a importância no crescimento destes estudos uma vez que o conteúdo de eletroquímica está entre os mais difíceis de ensinar e de aprender (NOGUEIRA; GOES; FERNANDEZ, 2017).

\subsection{Aspectos Metodológicos}

Na temática Aspectos Metodológicos considerou-se as seguintes categorias: a) Natureza da pesquisa; b) Abordagem da Pesquisa; c) Objetivo da Pesquisa e d) Nível de Ensino. Pode-se observar, de modo geral, que uma tendência das pesquisas sobre o ensino e aprendizagem de conceitos eletroquímicos, nas revistas investigadas, em relação a categoria Natureza da Pesquisa que $94,74 \%$ são pesquisas empíricas, tendo como principais instrumentos coleta de dados questionários, testes, entrevistas e exercícios. Ou seja, a maioria dos estudos voltam-se para a comprovação prática das teorias, através da realização de experimentos e de observações de determinados contextos para a coleta de dados no campo de estudo (CHIZZOTTI, 2018). E, os estudos teóricos relatam e discutem propostas ao ensino e aprendizagem de conceitos eletroquímicos, apresentando contribuições teóricas sobre o assunto.

Quanto à Abordagem de Pesquisa, conforme pode ser observado na Figura 3 há predominância $(89,47 \%)$ de investigações de caráter qualitativo dentre os artigos analisados. $E$, um dos artigos analisados desenvolveu uma abordagem de cunho quantitativo, isso aponta para uma necessidade de pesquisas acerca da aprendizagem de conceitos científicos que utilizem a complementariedade da abordagem quantitativa como um desenho de uma pesquisa mista, como forma de triangular os dados, analisar o fenômeno em estudo de uma maneira mais holística, mesmo que o peso da pesquisa ou preponderância seja da abordagem qualitativa (CRESWELL, 2007).

Figura 3. Categoria e subcategorias de análise relacionados aos aspectos metodológicos de pesquisas sobre ensino e aprendizagem de conceitos em eletroquímica.

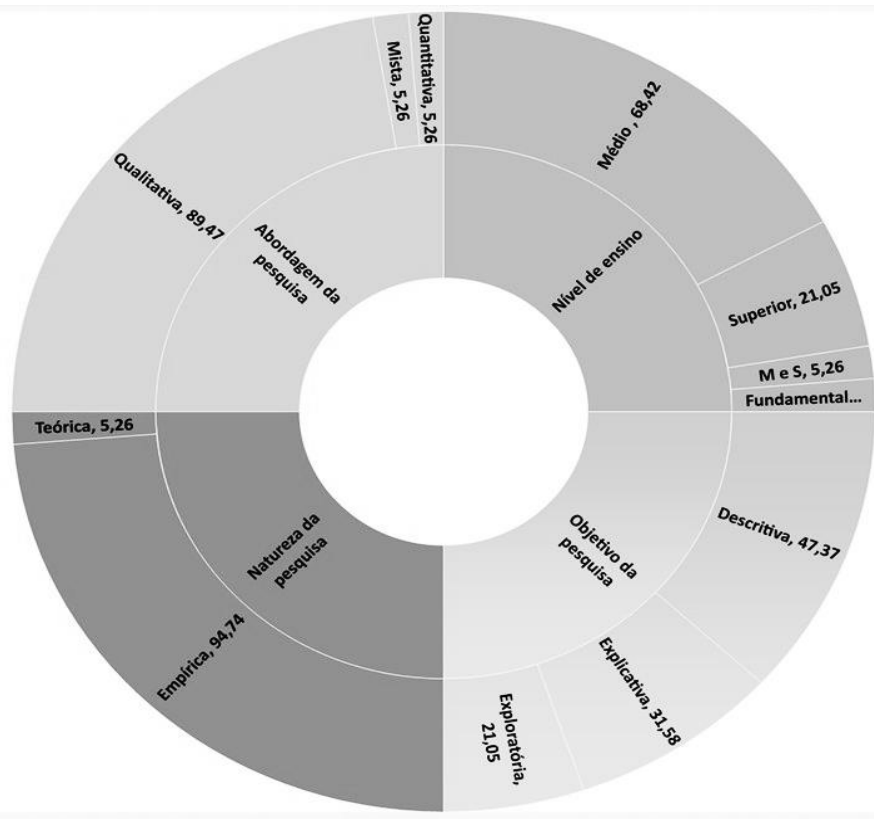

Fonte: Elaborada pelos autores. 
Quanto à categoria Objetivos da Pesquisa, observou-se que não existe diferença significativa entre as subcategorias Exploratória, Descritiva e Explicativa, mesmo que a maior predominância seja de pesquisas descritivas $47,37 \%$ (Figura 3). Os três tipos de pesquisa têm sua importância e depende tanto do fenômeno em estudo, quanto a maneira como se quer investigá-lo, sendo ainda, que esses tipos de pesquisa podem ser combinados e sequenciados (CRESWELL, 2007). Neste texto, optou-se por classificar os estudos considerando a característica mais forte em cada pesquisa.

Por fim, na categoria Nível de Ensino, verificou-se predominância de trabalhos voltados para as subcategorias Ensino Médio (68,42\%) e Educação Superior (21,05\%) demonstrando o forte interesse dos estudos nestes níveis de ensino. Estudos voltados a pesquisar o Ensino Fundamental e concomitantemente mais de um nível de ensino, como Ensino Médio e Ensino Superior, apresentaram menor percentual $(5,26 \%)$ significando o menor interesse das pesquisas por estudos deste tipo (Figura 3).

\subsection{Base Teórica de Fundamentação das Pesquisas}

Para essa temática foram considerados dois grandes campos do conhecimento, sendo um relacionado a Psicologia da Educação e outro à Didática das Ciências. Vale esclarecer que as categorias elaboradas não são excludentes, visto que a maioria das pesquisas (artigos), que fazem parte deste estudo, têm como aporte teórico os dois campos do conhecimento. Conforme mencionado anteriormente, as categorias relacionadas ao tema Base Teórica emergiram dos dados obtidos.

De modo geral, Psicologia da Educação tem como objetos os aspectos cognitivo, afetivo e psicomotor do professor e do aluno em situação educativa, conteúdos e estratégias de ensino e aprendizagem, utilizando métodos científicos para a investigação. Nesse sentido, as teorias de aprendizagem mais frequentes estão relacionadas ao Cognitivismo e Estudos de Aspectos Motivacionais (LEFRANÇOIS, 2009). Em relação à Didática das Ciências, esclarece-se que consiste em um campo do conhecimento que tem como centralidade questões específicas de ensino e de aprendizagem das Ciências Naturais - Biologia, Física e Química. Dessa maneira, esse campo de conhecimento visa articular saberes da História, Filosofia, Sociologia das Ciências com os da Educação para compreender como os alunos da Educação Básica e Superior interpretam certos fenômenos naturais, contextualizar a ideia de Ciência que os alunos construíram e sua humanização (CACHAPUZ et al., 2005).

Observando a Figura 4, pode-se inferir que nos anos 90 as pesquisas sobre ensino e aprendizagem de conceitos eletroquímicos estavam mais fundamentadas a partir da Didática das Ciências, tendo como principais contribuições: experimentação no ensino, seja demonstrativa e com alguns elementos investigativos; concepções alternativas; e obstáculos epistemológicos. Já no período de 2001 a 2010, os dois campos do conhecimento passam a ser utilizados concomitantes para orientar as pesquisas. Ainda sobre o referido período, é possível afirmar que os constructos teóricos mais frequentes na categoria Cognitivismo são relacionados à Teoria da Aprendizagem Significativa e da Teoria da Equilibração (Quadro 3). Em relação a Didática das Ciências, foram observados predominante pesquisas que abordavam concepções 
alternativas, e outras pesquisas em menor frequência, como: abordagem temática (1); experimentação (1); resolução de problemas (1); níveis do conhecimento químico (Quadro 3).

Figura 4. Principais construtos teóricos presentes nas pesquisas sobre ensino e aprendizagem de conceitos eletroquímicos.

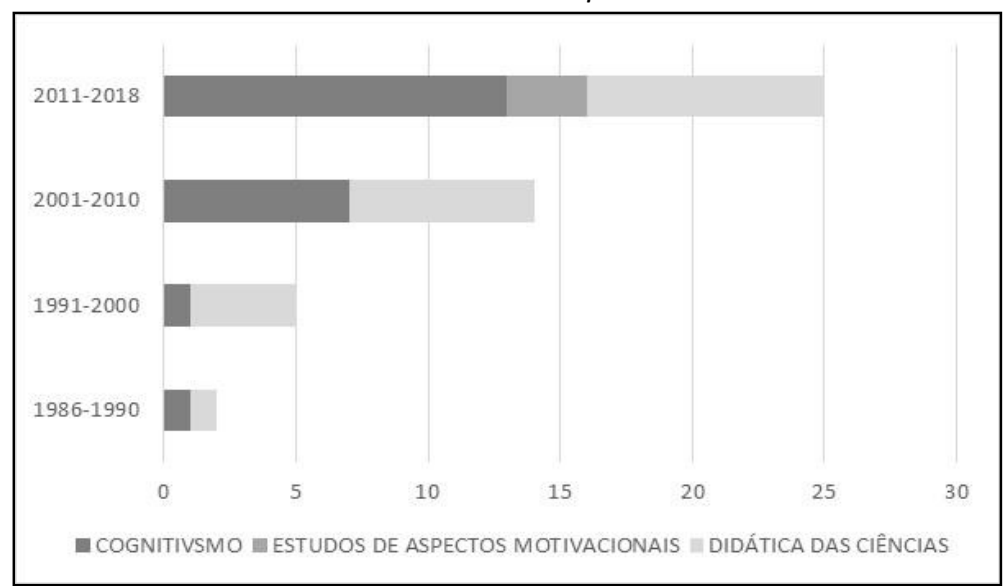

Fonte: Elaborada pelos autores.

No período seguinte, 2011 a 2018, são mais frequentes as pesquisas que abordam o ensino e aprendizagem de conceitos eletroquímicos, o embasamento teórico pautado em construtos da Psicologia da Educação. Além da categoria Cognitivismo também foram encontradas Estudos de Aspectos Motivacionais - motivação e engajamento escolar. Concernente à categoria Cognitivismo, além da Teoria da Aprendizagem Significativa, ganha destaque a Teoria Sociocultural. E ainda, uma pesquisa foi norteada pela Teoria dos Campos Conceituais e quatro outras pesquisas foram classificadas na subcategoria outros construtos, tais como: representações dos estudantes - um trabalho assume como representação mental e habilidades cognitivas. Em relação à Didática das Ciências, observou-se que a subcategoria mais frequente foi a Experimentação, mas foram analisadas duas outras pesquisas que abordavam concepções alternativas e níveis do conhecimento químico (Quadro 3).

Quadro 3. Bases teóricas presentes nas pesquisas sobre ensino e aprendizagem de conceitos eletroquímicos.

\begin{tabular}{|c|c|c|}
\hline CATEGORIAS & SUBCATEGORIAS & FREQUÊNCIA \\
\hline \multirow{5}{*}{ Cognitivismo } & Teoria da Aprendizagem Significativa & 6 \\
\hline & Teoria Sociocultural & 6 \\
\hline & Teoria da Equilibração & 3 \\
\hline & Teoria dos Campos Conceituais & 1 \\
\hline & Outros construtos & 6 \\
\hline \multirow{2}{*}{$\begin{array}{l}\text { Estudos de Aspectos } \\
\text { Motivacionais }\end{array}$} & Motivação & 1 \\
\hline & Engajamento escolar & 2 \\
\hline \multirow{6}{*}{ Didática das Ciências } & Concepções Alternativas & 5 \\
\hline & $\begin{array}{c}\text { Experimentação (demonstrativa, investigativa } \\
\text { e/ou problematizadora) }\end{array}$ & 11 \\
\hline & Obstáculos epistemológicos & 1 \\
\hline & Níveis do conhecimento químico & 2 \\
\hline & Abordagem temática & 1 \\
\hline & Resolução de problemas & 1 \\
\hline
\end{tabular}

Fonte: Elaborada pelos autores. 


\subsection{Resultados nas Pesquisas}

Quanto aos principais resultados apresentados nas pesquisas, foram sistematizados em duas categorias: Dificuldades de Aprendizagem mais recorrentes e Estratégias Remediativas das Dificuldades de Aprendizagem (Quadro 4).

Quadro 4. Análise dos resultados das pesquisas sobre ensino e aprendizagem de conceitos eletroquímicos.

\begin{tabular}{|c|c|c|c|}
\hline CATEGORIAS & & UNIDADES REPRESENTATIVAS & PESQUISAS \\
\hline \multirow{4}{*}{$\begin{array}{l}\text { Dificuldades de } \\
\text { aprendizagem }\end{array}$} & $\begin{array}{l}\text { Inapropriação } \\
\text { de conceitos } \\
\text { químicos }\end{array}$ & $\begin{array}{l}\text { (1) Uso inadequado de conceitos científicos e de } \\
\text { vocabulário específico da eletroquímica; (2) } \\
\text { Ausência dos conceitos necessários para } \\
\text { interpretação; (3) Dificuldades de desenvolver } \\
\text { argumentos para justificar suas respostas; (4) } \\
\text { Abordagem inadequada dos conceitos químicos } \\
\text { em livros didáticos. }\end{array}$ & \multirow{4}{*}{$\begin{array}{l}\text { GUISASOLA et al., } \\
\text { 2008; COSTA; } \\
\text { OLIVEIRA; ALVES, } \\
\text { 2008; CASSIO et } \\
\text { al., 2012; PACCA } \\
\text { et al., 2003; } \\
\text { LABURÚ; } \\
\text { GOUVEIA; } \\
\text { BARROS, 2009; } \\
\text { CARAMEL; PACCA } \\
\text { 2011; PESA et al., } \\
\text { 2014). }\end{array}$} \\
\hline & $\begin{array}{l}\text { Incompreensão } \\
\text { dos modelos } \\
\text { científicos }\end{array}$ & $\begin{array}{l}\text { (5) Incompreensão de modelos; (6) Não } \\
\text { constroem facilmente modelos científicos } \\
\text { explicativos; }\end{array}$ & \\
\hline & $\begin{array}{l}\text { Incompreensão } \\
\text { dos fenômenos }\end{array}$ & $\begin{array}{l}\text { (7) Limitação na explicação do comportamento } \\
\text { dos materiais; (8) Explicações macroscópicas aos } \\
\text { conceitos; (9) Incompreensão do papel dos íons } \\
\text { nas transformações químicas; }\end{array}$ & \\
\hline & $\begin{array}{l}\text { Não seguem } \\
\text { procedimentos }\end{array}$ & $\begin{array}{l}\text { (10) Falta de aplicação dos conhecimentos } \\
\text { teóricos no planejamento; (11) Dificuldades de } \\
\text { desenvolver um experimento; }\end{array}$ & \\
\hline \multirow[b]{2}{*}{$\begin{array}{l}\text { Estratégias } \\
\text { remediativas } \\
\text { das } \\
\text { dificuldades de } \\
\text { aprendizagem }\end{array}$} & $\begin{array}{l}\text { Processo de } \\
\text { construção dos } \\
\text { conceitos } \\
\text { científicos }\end{array}$ & $\begin{array}{l}\text { (1) Valorização e reconhecimento do } \\
\text { conhecimento prévio; } \\
\text { (2) Abordagem de temas sociais e } \\
\text { contextualizados; } \\
\text { (3) Abordagem dos três níveis de conhecimento; } \\
\text { (4) Construção dos conceitos ao longo da história } \\
\text { das ciências; }\end{array}$ & \multirow{2}{*}{$\begin{array}{l}\text { BOFF; FRISON, } \\
\text { 1996; LOPES, } \\
\text { 1996; FRAGAL et } \\
\text { al., 2011; } \\
\text { SANJUAN et al., } \\
\text { 2009; SILVA, et } \\
\text { al., 2016; } \\
\text { BARRETO; } \\
\text { BATISTA; CRUZ, } \\
\text { 2017; SANTOS, } \\
\text { 2018; COSTA; } \\
\text { OLIVEIRA; ALVES, } \\
\text { 2008; FRANCISCO } \\
\text { JUNIOR, 2013; } \\
\text { COELHO; } \\
\text { AMANTES, 2014; } \\
\text { SANTOS JUNIOR, } \\
\text { et al., 2016; } \\
\text { VIEIRA et al., } \\
\text { 1986; LABURÚ; } \\
\text { GOUVEIA; } \\
\text { BARROS, 2009. }\end{array}$} \\
\hline & $\begin{array}{l}\text { Atividades } \\
\text { didáticas } \\
\text { diferenciadas }\end{array}$ & $\begin{array}{l}\text { (5) Uso da experimentação demonstrativa, } \\
\text { investigativa e problematizadora; } \\
\text { (6) Uso da simulação por computador; } \\
\text { (7) Atividades problematizadoras; } \\
\text { (8) Uso do material didático aliado a discussões; } \\
\text { (9) Uso da argumentação na interação com colegas } \\
\text { e com o professor. } \\
\text { (10) Articulação das simbologias convencionais; } \\
\text { (11) Elaboração de desenhos }\end{array}$ & \\
\hline
\end{tabular}

Fonte: Elaborada pelos autores.

$\mathrm{Na}$ categoria Dificuldades de Aprendizagem, a inapropriação de conceitos científicos aparece nas pesquisas com o uso inadequado de conceitos químicos e/ou com a ausência de vocabulário específico da eletroquímica e a dificuldade de argumentar e justificar suas respostas. Essa dificuldade está relacionada a natureza microscópica e por vezes abstrata do conhecimento químico e por ser a linguagem química basicamente simbólica, o que exige alta capacidade de abstração e generalização do conhecimento. Corroborando, Mortimer (2011, p. 187) afirma que “[...] a linguagem cotidiana é automática e muito mais próxima da fala [...] a 
linguagem científica exige uma reflexão consciente no seu uso [...]". E isto, pode interferir na apropriação dos conceitos científicos pelos alunos, uma vez que estes ainda são um recurso muito utilizado para nortear seus estudos.

As pesquisas identificaram estudantes com limitação na explicação do comportamento dos materiais, com tendência a dar explicações macroscópicas aos conceitos e que não compreendem o papel dos íons nas transformações químicas. A literatura destaca que conceitos como carga, diferença de potencial e capacidade elétrica, redução, corrente elétrica, condutibilidade elétrica em soluções, representação de reações redox, potencial de redução, dependência entre as reações de oxidação e redução, processo de transferência de elétrons, significado do número de oxidação, identificação dos reagentes em oxidantes e redutores, identificação das reações redox e o balanceamento das reações de oxirredução (GARNETT; TREAGUST, 1992a; 1992b; DE JONG; TREAGUST, 2002), não são utilizados pela maioria dos estudantes em suas interpretações, configurando dessa maneira que os alunos apresentam dificuldade na diferenciação das reações no nível macroscópico e no nível microscópico (BARKE; HAZARI; YITBAREK, 2009).

Vale ressaltar que, as dificuldades verificadas nas pesquisas consistem em problemas persistentes no aprendizado de eletroquímica, como por exemplo a determinação dos polos das pilhas (MATSUBARA; NERI; ROSOLEN, 2007), a incompreensão de reações químicas e reações redox (MORTIMER; MIRANDA, 1995; JUSTI, 1998) e a abordagem inadequada destes conceitos em Livros Didáticos (MENDONÇA; CAMPOS; JÓFILI, 2004; PITANGA et al., 2014).

$\mathrm{Na}$ categoria Estratégias Remediativas das Dificuldades de Aprendizagem foram incluídas pesquisas que mostram métodos e atividades que promovem a efetiva aprendizagem. Observou-se que, as atividades didáticas propostas foram organizadas tendo em vista o processo de construção dos conceitos científicos, partindo do diagnóstico por meio da valorização e reconhecimento do conhecimento prévio dos alunos e, organizando atividades em abordagens de temas sociais e contextualizados, a partir da valoração da realidade cotidiana do estudante, como forma de motivar e despertar o interesse pela Ciência.

Destaca-se ainda, atividades didáticas diferenciadas como a experimentação demonstrativa, investigativa e problematizadora, que configuram como estratégias facilitadoras da aprendizagem de conceitos. Wartha e Lemos (2016) destacam que os momentos de discussões, podem ajudar os estudantes a relacionarem o conceito com o contexto interno e externo a realidade escolar e a participação na atividade de forma intelectualmente ativa. Em relação ao uso da experimentação e da simulação por computador, algumas pesquisas mencionam que o rendimento dos alunos aumenta, destacando a importância da ação docente na evolução do aprendizado, pois conforme Santos e Greca (2005) alunos com dificuldades de gerar representações químicas foram os mais beneficiados pelo uso da simulação. Isto porque as simulações colaboram na visualização dos fenômenos no nível micro e submicroscópico.

Outra atividade didática diferenciada consiste na elaboração de desenhos, sendo mencionada estratégias eficazes quanto de ensino de conceitos científicos e no diagnóstico de concepções e modelos dos estudantes. Todavia, o uso de imagens ainda é restrito em sala de aula, e quando ocorre geralmente é por meio das imagens presentes nos livros didáticos, os 
quais prezam mais por imagens explicativas, de um fenômeno ou estrutura e sendo os cientistas, na maioria das vezes, somente citados pelo nome, resultando no desconhecimento do cientista pela imagem. Por fim, vale acrescentar que nem todas as atividades são igualmente eficazes para se conseguir qualquer tipo de aprendizagem. Segundo Pozo (2002) a realização de diversas atividades de aprendizagem com o fim de promover a reflexão sobre o que se está aprendendo (reflexiva) é muito mais lucrativa.

\section{Considerações Finais}

O estudo realizado possibilitou observar a predominância de pesquisas empíricas e de caráter qualitativo, tendo como principais instrumentos coleta de dados questionários, testes, entrevistas e exercícios. Essas pesquisas assumem aportes teóricos de dois grandes campos do conhecimento, a Psicologia da Educação e a Didática das Ciências. Além da Base Teórica que fundamenta as pesquisas as principais contribuições das investigações referem-se as dificuldades de aprendizagem mais recorrentes e as estratégias remediativas dessas dificuldades de aprendizagem. Neles constam as dificuldades em compreender os conceitos eletroquímicos e as estratégias didáticas para superar essas limitações na sala de aula.

Vale destacar que os resultados mostram que a ausência de discussões sobre ciência nas aulas reforça a falta de valorização das relações existentes entre teorias e modelos científicos, que tentam explicar a natureza da matéria e os fenômenos observáveis. Nisto, as estratégias remediativas apresentadas nas pesquisas têm a finalidade de superação das dificuldades de aprendizagem, sendo possibilidades de desenvolvimento da aprendizagem de conceitos em eletroquímica. A articulação das simbologias convencionais e a elaboração de desenhos são atividades diferenciadas que colaboram na compreensão de conceitos complexos como os de eletroquímica.

Percebe-se ainda algumas limitações como a exploração dos modelos didáticos na compreensão de modelos científicos próprios da eletroquímica, como também lacunas a serem investigadas no estudo dos modelos mentais dos estudantes durante a aquisição de conceitos eletroquímicos.

\section{Agradecimentos e Apoio}

À Fapeam pela concessão da bolsa.

\section{Referências}

ALVES-MAZZOTTI, A. J.; GEWANDSZNAJDER, F. O método nas ciências naturais e sociais: pesquisa quantitativa e qualitativa. 2 ed., São Paulo: Pioneira, 1999.

BARKE, H.; HAZARI, A.; YITBAREK, S. Misconceptions in Chemistry: Addressing Perceptions in Chemical Education. Springer-Verlag Berlin Heidelberg. Berlim: Springer. n. 1, p. 11-294, 2009.

BERLOTTI, M. Dificuldades conceituais no aprendizado de equilíbrios químicos envolvendo reações ácido-base. Química Nova. São Paulo, v. 34, n. 10, p. 1836-1839, 2011. 
CACHAPUZ, A.; GIL-PEREZ, D.; CARVALHO, A. M. P.; PRAIA, J.; VILCHES, A. A necessária renovação do Ensino de Ciências. São Paulo: Cortez, 2005.

CHIZZOTTI, A. Pesquisa em ciências humanas e sociais. 12 ed. São Paulo: Cortez, 2018.

COSTA, S. S. C.; MOREIRA, J. P. Resolução de problemas I: diferenças entre novatos e especialistas. Investigações em Ensino de Ciências. Porto Alegre, v.1, n.2,176-192, 1996.

CRESWELL, J. W. Projeto de pesquisa: método qualitativo, quantitativo e misto. Tradução de Luciana Ferreira da Rocha. 2 ed., Porto Alegre: Artmed, 2007.

DEMO, Pedro. Metodologia do conhecimento científico. São Paulo: Atlas, 2000.

DE JONG, O.; TREAGUST, D. F. The teaching and learning of electrochemistry. In: J. K. Gilbert; O. De Jong; R. Justi; D. F. Treagust e J. H. Van Driel (Eds.), Chemical Education: Towards Researchbased Practice Dordrecht: Kluwer Academic Publishers, p. 317-337, 2002.

FLICK, U. Introdução a pesquisa qualitativa. Tradução de Joice Elias Costa. 3 ed. Porto Alegre: Artmed, 2009.

FONSECA, J. J. S. Metodologia da Pesquisa Científica. Apostila do Curso de especialização em comunidades virtuais de aprendizagem da Universidade Estadual do Ceará, 2002. Disponível em: http://www.ia.ufrrj.br/ppgea/conteudo/conteudo-2012-

1/1SF/Sandra/apostilaMetodologia.pdf. Acesso em: 09 nov. 2019.

FRANCISCO JUNIOR, W. E.; DOCHI, R. S. Um experimento simples envolvendo óxido-redução e diferença de pressão com Materiais do dia a dia. Química Nova na Escola, v. 23, 2006.

FRANCISCO JUNIOR, W. E.; FERREIRA, L. H.; HARTWIG, D. R. Um modelo para o estudo de deposição metálica. Química Nova na Escola. v. 31, n. 2, 2009.

FRISON, M. D.; DEL PINO, J. C. ENSINO E APRENDIZAGEM EM CIÊNCIAS: MANIFESTAÇÕES DE ESTUDANTES SOBRE O AMBIENTE ESCOLAR. Ensino de Ciências e Tecnologia em Revista, v. 5, n. 2, p. 35-46, 2015.

GARNETT, J.; TREAGUST, D.F. Conceptual difficulties experienced by senior high school students of electrochemistry: electric circuits and oxidation-reduction equations. Journal of Research in Science Teaching. Berlim, v. 29, n. 2, p. 121-142, 1992a.

. J.; TREAGUST, D.F. Conceptual difficulties experienced by senior high school students of electrochemistry: Electrochemical (galvanic) and electrolityc cells. Journal of Research in Science Teaching. Berlim, v. 29, n. 10, p. 1079-1099, 1992b.

GERHARDT, T. E.; SILVEIRA, D. T. Métodos de pesquisa. Porto Alegre: Editora da UFRGS, 2009.

GERMANO, M. G.; LIMA, I. P. C. de; SILVA, A. P. B; da. Pilha voltaica: entre rãs, acasos e necessidades. Caderno Brasileiro de Ensino de Física, v. 29, n. 1: p. 145-155, 2012.

GIL, A. C. Como elaborar projetos de pesquisa. 4 ed. São Paulo: Atlas, 2002.

Métodos e técnicas de pesquisa social. 6 ed. São Paulo: Atlas, 2008.

HOPPEN, N., MEIRELLES, F. S. Sistemas de Informação: um panorama da pesquisa científica entre 1990 e 2003. Revista de Administração de Empresas, v. 45, n.1, 24-35, 2005. 
JUSTI, R. S. A afinidade entre as substâncias: pode explicar as reações químicas? Química Nova na Escola. n. 7, 1998.

KLEIN, S. G.; BRAIBANTE, M. E. F. Reações de oxi-redução e suas diferentes abordagens. Química Nova na Escola. v. 39, n. 1, p. 35-45, 2017.

LEFRANÇOIS, G. R.; Teorias de Aprendizagem: O que a velha senhora disse. 5. ed. São Paulo: Cengage Learning, 2009.

LIMA, J. O. G e LEITE, L. R. O processo de ensino e aprendizagem da disciplina de Química: o caso das escolas do ensino médio de Crateús/Ceará/Brasil. Revista Electrónica de Investigación em Educación em Ciencias, v.7, n.7, p.72-85, 2012.

LIMA, V. F.; MERÇON, F. Metais Pesados no Ensino de Química. Química Nova na Escola. v. 33, n. 4, p. 199-205, 2011.

MATHIAS, L. C.; SILVA, P. R. C. D; SILVA, O. H. M. D. Um instrumento alternativo ao estudo de pilhas recarregáveis via Arduino. Caderno Brasileiro de Ensino de Física, Paraná, v. 34, n. 1, p. 310-323, 2017.

MATSUBARA, E. Y.; NERI, C. R.; ROSOLEN, J. M. Pilhas Alcalinas: um dispositivo útil para o ensino de Química. Química Nova, São Paulo, v. 30, n. 4, p. 1020-1025, 2007. Disponível em: http://www.scielo.br/pdf/qn/v30n4/a46v30n4.pdf. Acesso em: 30 out. 2019.

MENDONÇA, R. J.; CAMPOS, A. F.; JÓFILI, Z. M. S. O conceito de oxidação-redução nos livros didáticos de química orgânica do Ensino Médio. Química Nova na Escola. v. 20, n. 20, p. 45-48, 2004.

MERÇON, F.; GUIMARÃES, P. I. C; MAINIER, F. B. Corrosão: um fenômeno usual de fenômeno químico. Química Nova na Escola. v. 19, n. 1, p. 11-14, 2004.

MORAES, R.; GALIAZZI, M. D. C. Análise Textual Discursiva. Ijuí: Unijuí, 2007.

MORTIMER, E. F. As chamas e os Cristais Revisitados: estabelecendo diálogos entre a linguagem cintífica e a linguagem cotidiana no ensino das Ciências da natureza. In: Santos W.L.P. e Maldaner, O. A. Ensino de química em foco. Capítulo 7, p. 183 - 207, 2011.

MORTIMER, E. F.; MIRANDA, L. C. Transformações: Concepções de estudantes sobre reações químicas. Química Nova na Escola, n.2, novembro, 1995.

.Transformações: Concepções dos estudantes sobre reações químicas. Química Nova na

Escola. Minas Gerais, n. 2, p. 23-26, 1999. Disponível em: http://qnesc.sbq.org.br/online/qnesc02/aluno.pdf. Acesso em: 31 out. 2019.

NOGUEIRA, K. S. C; GOES, L. F. D; FERNANDEZ, C. O estado da arte sobre o ensino de reações redox nos principais eventos na área de educação no Brasil. Revista Electrónica de Enseñanza de las Ciencias, v. 16, n. 3, p. 410-434, 2017. Disponível em: http://reec.uvigo. es/volumenes/volumen16/REEC_16_3_1_ex1106.pdf. Acesso em: 31 out. 2019.

OLIVEIRA, M. M. Como fazer pesquisa qualitativa. Petrópolis/RJ: Editora Vozes, 2007. 
PITANGA, A. F.; SANTOS, H. B.; GUEDES, J. T.; FERREIRA, W. M.; SANTOS, L. D. História da Ciência nos Livros Didáticos de Química: Eletroquímica como Objeto de Investigação. Química Nova na Escola. São Paulo, v. 36, n. 1, p. 11-17, 2014.

POZO, J. I. Aprendizes e mestres: a nova cultura da aprendizagem. Porto Alegre: Artmed, 2002. RODRÍGUEZ, L. G.; PÉREZ, B. C. Aprendizaje de las reacciones químicas a través de actividades de indagación en el laboratorio sobre cuestiones de la vida cotidiana. Enseñanza de las ciências. Santiago de Compostela, v. 34, n. 3, p. 143-160, 2016.

ROSA, M. I. F. P. S; SCHNETZLER, R. P. Sobre a importância do conceito transformação química no processo de aquisição do conhecimento químico: subtítulo do artigo. Química Nova na Escola: Pesquisa no Ensino de Química, v. 1, n. 8, p. 31-35, 1998. Disponível em: http://www.contagem.mg.gov.br/arquivos/comunicacao/femcitec_sobreoconceitodatransfor macao09.pdf. Acesso em: 1 nov. 2019.

SANTOS, F. M. T; GRECA, I. M. Promovendo aprendizagem de conceitos e de representações pictóricas em Química com uma ferramenta de simulação computacional. Revista Electrónica de Enseñanza de las Ciencias. v. 4, n. 1, 2005.

SARTORI, E. R.; BATISTA, E. F.; FATIBELLO-FILHO, O. Escurecimento e Limpeza de Objetos de Prata: Um Experimento Simples e de Fácil Execução Envolvendo Reações de OxidaçãoRedução. Química Nova na Escola. n. 30, p. 107-111, 2008.

SARTORI, E. R.; SANTOS, V. B.; TRENCH, A. B.; FATIBELLO-FILHO, O. Construção de Uma Célula Eletrolítica para o Ensino de Eletrólise a Partir de Materiais de Baixo Custo. Química Nova na Escola. v. 35, n. 2, p. 107-111, 2013.

UEHARA, F. M. G. Refletindo dificuldades de alunos do Ensino Médio no estudo do Equilíbrio Químico. Dissertação (Mestrado em Ensino de Ciências e Matemática) - Universidade Federal do Rio Grande do Norte. Natal, RN, 2005.

WARTHA, E. J.; LEMOS, M. M. Abordagens investigativas no ensino de Química: limites e possibilidades. Amazônia, v. 12, n. 24, p. 5-13, 2016.

WARTHA, E. J.; REIS, M.S.; SILVEIRA, M. P.; GUZZI FILHO, N. J.; JESUS, R. M. A maresia no ensino de Química. Química Nova na Escola. n. 26, p. 17-20, 2007. 